\title{
U tilidad clínica de la fusión SPECT/CT con marcadores externos en patología oncológica
}

\author{
Carolina Aqueveque $A^{1}$, Patricio González $E^{1}$, Daniela \\ G utiérrez $\mathrm{B}^{1}$, Rodrigo Jaimovich $\mathrm{F}^{1}$, Juan Carlos Díaz $\mathrm{P}^{2}$, \\ Paula Csendes $G^{2}$, Patricia 0 rellana $P^{2}$, Hugo Lavados $M^{1}$, \\ Isabel Alliende $\mathrm{G}^{\mathrm{Ia}}$, Sandra Araya $\mathrm{L}^{2 \mathrm{a}}$. \\ Fusion of SPECT with computed \\ tomography or magnetic resonance \\ for the interpretation of abnormal \\ tracer uptake
}

Background: Interpretation of abnormal foci with high tracer uptake may require morphological correlation. Fusion of functional images obtained by single photon emission computed tomography (SPECT) and anatomical images obtained by computed tomography (CT) or magnetic resonance (RM) allows an integrated comprehension of complementary information. Aim: To demonstrate that SPECT/CT fusion with external markers is useful in clinical practice to clarify the location and pathological meaning of questionable foci. Material and methods: Thirty four pairs of images from separate equipments (31 SPECT/CT and 3 SPECT/RM) pertaining to 29 patients, were fused. Fifty one foci of abnormal tracer uptake of uncertain pathological meaning were analyzed. These were classified before and after the fusion as probably malignant or probably benign. Results: Seventy percent of patients had a differentiated thyroid carcinoma. The fusion localized $100 \%$ of foci. Nine percent had a normal and $26 \%$ an abnormal anatomy. Before fusion $82 \%$ of foci were classified as potentially malignant. This figure changed to $59 \%$ after the fusion ( $\mathrm{p} \varangle 0.01$ ). Therefore the suspicion of malignancy was presumptively confirmed in $72 \%$ of foci and fusion results would have reached a $27 \%$ of incremental diagnostic value in 14 cases that changed of category (11 with differentiated thyroid carcinoma, one with colorectal cancer, one with a nasal Ewing sarcoma and one with a brain tumor). Conclusions: The fusion of SPECT and CT is useful in selected patients, specially those with differentiated thyroid carcinoma. The fusion of SPECT and RM is also feasible (Rev Méd Chile 2007; 135: 725-34).

(Key words: Magnetic resonance imaging; Thyroid neoplasms; Tomography emission-computed, Single-photon)

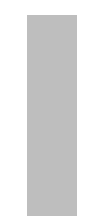

\footnotetext{
Recibido el 23 de marzo, 2006. Aceptado el 20 de noviembre, 2006.

Proyecto OAIC 037/04 financiado por la Oficina de Apoyo a la Investigación Clínica (OAIC) y la Sección de Medicina Nuclear del Hospital Clínico de la Universidad de Chile.

${ }^{1}$ Sección Medicina Nuclear, Departamento de Medicina, Hospital Clínico Universidad de Chile. ${ }^{2}$ Centro de Imagenología, Hospital Clínico Universidad de Chile.

aTecnólogo Médico.
}

Correspondencia a: Prof. Dr. Patricio González Espinoza. Santos Dumontt \# $9991^{\circ}$ piso E. Sección Medicina Nuclear, Hospital Clínico de la Universidad de Chile. Fono: 7770569 - 9788184 9788185. Fax: 7777618. E mail: ajgonzalez@manquehue.net 
T as imágenes en medicina nuclear y radiología _son complementarias y su fusión permitiría una comprensión integrada, respondiendo a la necesidad de entregar al clínico un formato amigable y confiable de la información ${ }^{1-4}$.

Para SPECT (tomografía por emisión de fotón único) o PET (tomografía por emisión de positrones), distintos radiotrazadores (RT) administrados al paciente revelan el estado funcional de órganos y tumores.

En oncología, los RT más utilizados son: $\mathrm{Tc}^{99 \mathrm{~m}}$ MDP (actividad osteoblástica en metástasis óseas), Iodo ${ }^{131}$ (en cáncer diferenciado tiroideo), Galio ${ }^{67}$ (tejido linfomatoso) y MIBG-131 (tumores neuroendocrinos). Para PET, Chile dispone de fluordeoxiglucosa (F18-FDG) para evaluación del metabolismo glucosídico ${ }^{5-7}$.

Por la relativa carencia de referentes anatómicos, SPECT y PET pueden requerir correlación morfológica dirigida. Su fusión con una tomografía computarizada (CT) o resonancia magnética (RM), constituye una pareja de imágenes de distinta modalidad; SPECT/CT, SPECT/RM, PET/ CT o PET/RM, capaz de localizar focos isotópicos en su estructura correspondiente, que puede 0 no estar alterada ${ }^{8-12}$.

La fusión con SPECT debiera lograr resultados similares en sistemas híbridos o separados, ya que comparten el mismo software y puede optimizarse con marcadores externos (ME) o internos (MI) al paciente ${ }^{13-16}$

Las ventajas y desventajas respectivas son discutidas en la literatura, principalmente atribuidas al alto costo y escasa disponibilidad de los híbridos y al consumo de tiempo en los separados. Sin embargo, consistentemente benefician a pacientes oncológicos, quienes en distintas etapas de su enfermedad requieren evaluación diagnóstica no invasiva que podría modificar la terapia ${ }^{5,6}$.

La revisión de 279 exploraciones sistémicas con radioyodo (ES-I ${ }^{131}$ ) realizada en el Hospital Clínico de la Universidad de Chile (periodo 2005), mostró que la correlación morfológica de los hallazgos fue requerida en $43,7 \%$ de los reportes; de los cuales $20,4 \%$ concluían enfermedad locorregional y $23,3 \%$ compromiso secundario extracervical. Los primeros presentaron agrupaciones de hasta 5 focos yodocaptantes y, al menos uno era de gran tamaño e intensidad y estaba ubicado en el tercio cervical superior o lateral. Esta experiencia fue extraída de la tesis para optar al título de especialista en medicina nuclear del primer autor y permitió desarrollar un algoritmo de selección de pacientes con cáncer diferenciado tiroideo (CDT) para fusión SPECT-I ${ }^{131} / \mathrm{CT}$ (Figura 1).

Ambos grupos fundamentan por frecuencia e implicancia terapéutica, la necesidad de desarrollar en el medio local un sistema de correlación anatómica más precisa que la actualmente disponible, cual es la comparación visual de la ES planar de cuerpo entero con CT o RM segmentario 15,17 .

La búsqueda de los pacientes, patologías y estudios nucleares que más se beneficiarían de la fusión, guían a su vez la búsqueda de la aplicación clínica más eficiente y necesaria, teniendo como objetivo determinar el significado patológico de focos isotópicos anormales de difícil localización para demostrar la utilidad de fusión SPECT/CT en equipos separados, con marcadores externos.

\section{MATERIAL Y MÉTODOS}

Estudio prospectivo-descriptivo desarrollado en la Sección de Medicina Nuclear (MN) y colaboración del Centro de Imagenología del Hospital Clínico de la Universidad de Chile.

Pacientes. Se reclutaron 34 pacientes que consultaron espontáneamente a MN entre abril 2004 y enero 2006: 22 mujeres, edad 38 \pm 16 (rango 5-68 años).

- Criterios de inclusión: Diagnóstico de referencia oncológico. Presencia de focos hipercaptantes anormales en el cintigrama solicitado por el médico tratante, no explicables por biodistribución habitual del RT o contaminación, cuya localización o significado patológico fue incierto, requiriendo correlación morfológica a juicio del médico nuclear.

- Criterios de exclusión: embarazo, lactancia, trastornos del movimiento o claustrofobia.

Todos firmaron consentimiento informado aprobado por el Comité de Ética del hospital y en menores de edad, firmaron sus padres.

Adquisición de imágenes y fusión. Los cintigramas fueron realizados según fueron solicitados sin modificaciones. Los seleccionados se complemeta- 


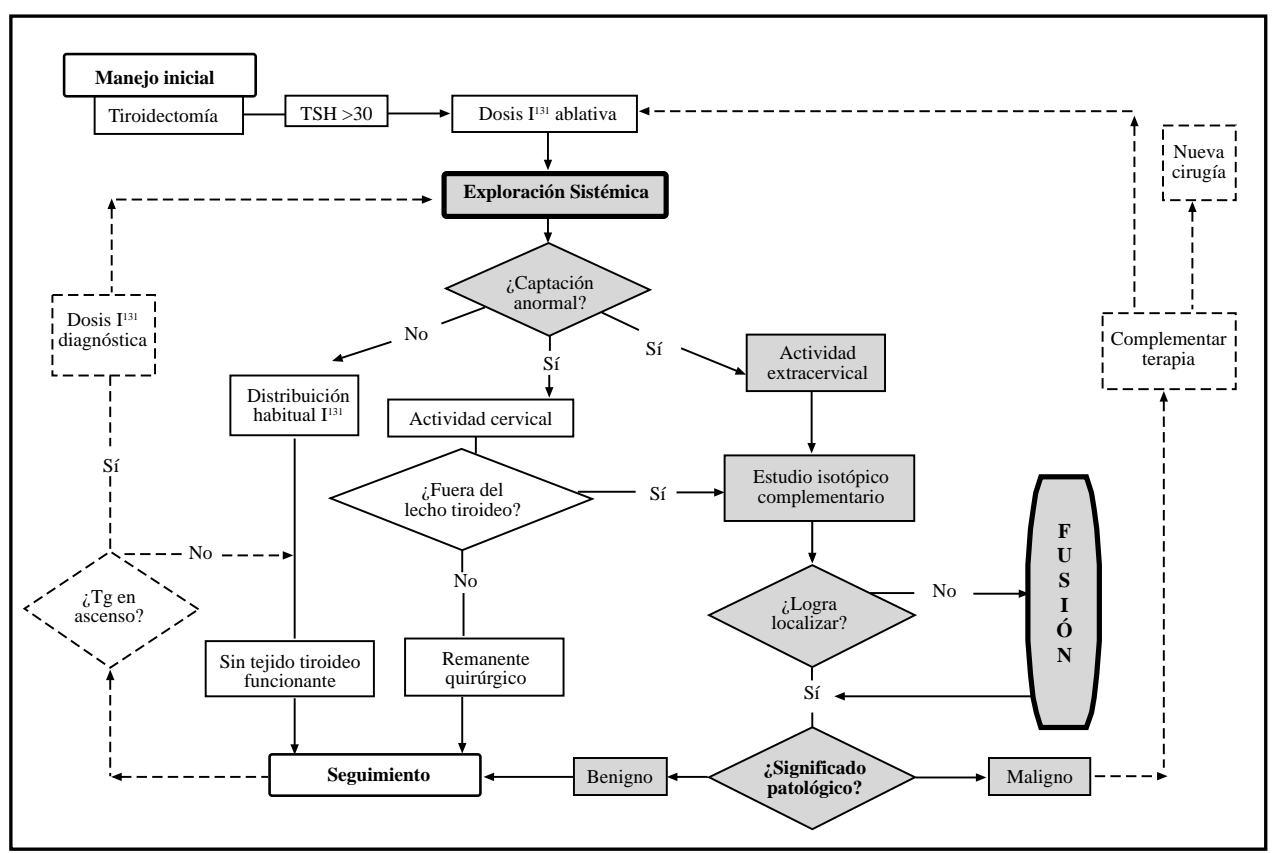

Figura 1. Criterio clínico de selección de pacientes con CDT para fusión (extraído de «Tesis para optar al título de especialista en medicina nuclear» Autor: Dra. Carolina Aqueveque. Disponible en Biblioteca Sección Medicina Nuclear, Hospital Clínico Universidad de Chile.

ron con SPECT sin RT adicional, portando 3 a $6 \mathrm{ME}$; en una gamacámara Siemens E.CAM 2002-doble cabezal. El mismo día se programó un CT dirigido (Siemens Somatom Duo 2002-dos canales), sin contraste yodado, portando los mismos ME.

Los pacientes debieron reproducir la posición bajo SPECT y CT apoyados por marcas cutáneas con tinta y supervisión de personal entrenado.

El protocolo local de CT fue modificado en cabeza-cuello al no angular el marco; en tórax donde la respiración fue espontánea; y en pelvis donde se mantuvo la vejiga semi-llena.

Excepcionalmente, se requirió RM (Siemens Magnetom Symphony 1.5 Tesla).

Todas las imágenes se adquirieron en formato DICOM.

La fusión y alineación tridimensional (ejes $\mathrm{x}, \mathrm{y}$, z) fue guiada por ME y MI, según el caso, en la estación de trabajo Siemens E.CAM, Software 3D E.Soft 3.5, ajustando las matrices a $128 \times 128$.

Marcadores externos. Se utilizó anillos radiopacos de $5 \mathrm{~mm}$ de diámetro marcados con $<1 \mu \mathrm{Ci} \mathrm{I}^{131} \mathrm{o}$ $\mathrm{Tc}^{99 \mathrm{~m}}$, detectables por CT y SPECT, que el paciente portó adheridos directamente a su piel en ambos estudios, sobre apófisis posteriores vertebrales, horquilla esternal, apéndice xifoides, espacios intercostales, espinas ilíacas ántero-superiores (EIAS), coxis y pubis, dependiendo del segmento en estudio.

Análisis de datos. Cada modalidad de imagen fue interpretada por un especialista en forma ciega, registrando independientemente los resultados cintigráficos planares, del CT y RM según aspecto, intensidad, ubicación y tamaño de los hallazgos. La interpretación de cada fusión SPECT/CT y SPECT/RM fue obtenida en conjunto por nuclear y radiólogo.

El aporte adicional de la fusión por sobre el cintigrama inicial, se obtuvo tras asignar a los focos una localización y significado patológico probable inicial y post fusión. Los focos de aspecto secundario, localizados o no, fueron considerados probablemente malignos (PM) y los de aspecto benigno con alta sospecha de su localización, en probablemente benigno (PB).

Adicionalmente, se obtuvo sensibilidad, especificidad, valor predictivo positivo (VPP) y negati- 
vo (VPN) de los cintigramas iniciales con test de Fisher, considerando la fusión como estándar de oro y la clasificación PM y PB como resultados positivos y negativos respectivamente.

\section{Resultados}

Se excluyeron 5/34 pacientes debido a inaplicabilidad del software de fusión por incumplimientos al protocolo; angulación del marco del CT $(\mathrm{n}=2)$, cantidad insuficiente de trazador en $\mathrm{ME}(\mathrm{n}=1)$ e incompatibilidad de segmentos adquiridos $(\mathrm{n}=2)$.

Se analizaron 29 pacientes, con 51 focos isotópicos, a partir de 24 exploraciones sistémicas$\mathrm{I}^{131}, 6$ cintigramas óseos-MDP-Tc ${ }^{99 m}, 1$ octreoscan-In ${ }^{111}, 2$ cintigramas MBG-I ${ }^{131}$ y 1 cintigrama Galio ${ }^{67}$-citrato. Se obtuvieron 34 fusiones (31 SPECT/CT y 3 SPECT/RM), con anatomía normal en 9 y alterada en 25 . El diagnóstico de referencia fue CDT en $70 \%$ de los casos.

Los ME fueron reemplazados por MI en estudios con MDP-Tc ${ }^{99 m}$, ya que la actividad osteoblástica esquelética sirvió de referente anatómico, y cuando la fusión fue órgano-dirigida al hígado (Figuras 2 y 3 ).

El significado patológico con las imágenes iniciales fue PM en $82 \%$ de los focos y PB en $18 \%$. La Figura 5 muestra que tras la fusión, estos porcentajes cambiaron a $59 \%$ y $41 \%$, respectivamente, alcanzando una diferencia estadísticamente significativa ( $\mathrm{p}=0,0021$ ).

La sospecha de malignidad y benignidad fue confirmada, presuntivamente, con la fusión en $72,5 \%$ de los casos, tras localizar 37/51 focos (Tabla 1). En cambio, el significado patológico cambió en 14/51 focos, afectando a 11 casos con CDT, 1 cáncer colo-rectal, 1 sarcoma de Ewing nasal y 1 tumor cerebral. Destaca el caso 31, en que un foco $\mathrm{PB}$ pasó a PM al no encontrarse correlato anatómico de la actividad mediastínica en una ES-I ${ }^{131}$, siendo considerada micrometástasis de CDT. El resto de los focos inicialmente clasificados PB mantuvieron esa categoría tras la fusión (Tabla 2).

La Figura 4 detalla el único caso cuya malignidad del foco fusionado fue confirmada histológicamente.

Así, los resultados de fusión habrían alcanzado $27 \%$ de valor diagnóstico incremental sobre el estudio inicial (cintigrama no fusionado) de ser confirmados por seguimiento o biopsia.

Con la condición de considerar la fusión como estándar de oro, los cintigramas iniciales obtendrían una sensibilidad 97\%, especificidad 38\%, VPP 69\% y VPN 89\% para diferenciar malignidad de benignidad, aun careciendo de localización exacta.

\section{DisCUSIÓN}

La correlación funcional/anatómica de imágenes es frecuentemente requerida en oncología en distintas etapas: diagnóstico, estadificación, evaluación de respuesta a tratamiento, seguimiento y sospecha de recurrencia ${ }^{5,6,18-20}$.

Las experiencias multicéntricas han evaluado diversos sistemas de fusión para oncología, destacando la trayectoria de Israel y col en equipos híbridos SPECT/CT monocabezal ${ }^{5,6,17,21}$, aún no disponibles en Chile.

Reconocer ventajas y desventajas de la fusión en equipos separados es indispensable para implementar un protocolo estandarizado de adquisición y procesamiento.

La principal dificultad radica en el posicionamiento del paciente durante ambos exámenes, la que está ausente en equipos híbridos, ya que SPECT y CT están acoplados en un mismo marco. Sin embargo, los movimientos respiratorios, peristálticos y llene vesical, se presentan tanto en híbridos como en separados, debido a la duración del SPECT (20-30 min). En consecuencia, los estudios para fusión son adquiridos en ciclo respiratorio libre y vejiga semi-llena.

El protocolo presentado incorporó marcadores que han sido avalados por otros estudios. Gutman comparó SPECT/CT-tórax con ME y MI en ciclo respiratorio normal e inspiración profunda y encontró mejor coincidencia entre ME en respiración libre que en inspiración (desfase 6-22 mm), observando un punto crítico en $4 \mathrm{ME}^{13}$. Los resultados fueron óptimos con ME localizados en horquilla esternal y columna vertebral, incluyendo sacro-coxis, debido a la reproducibilidad de posición del esqueleto axial bajo las camillas de gammacámara y tomógrafo. En cambio, la distinta convexidad de ellas favorece la rotación en cintura escapular y pelviana, generando desplazamiento de los ME en apófisis coracoides y EIAS. 


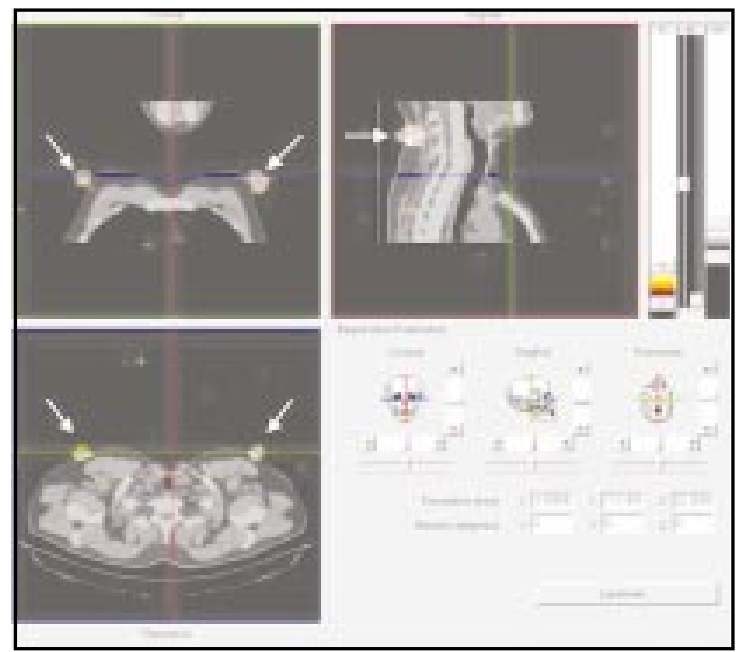

Figura 2. Pantalla de fusión 3D SPECT/CT mostrando marcadores externos radioopacos con $\mathrm{I}^{131}$ en región pectoral y columna cervical, visibles en los 3 ejes ortogonales (flechas), evidentes en ambos estudios.

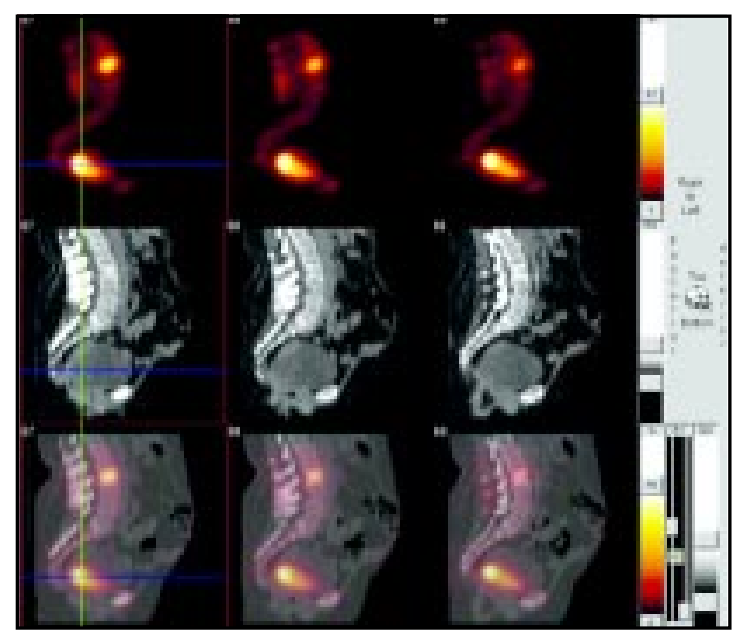

Figura 3. Caso 14. Mujer, 60 años. Cáncer rectal tratado (operación de Miles) en re-estadificación.

A. SPECT óseo Tc ${ }^{99 m}$ MDP muestra actividad osteoblástica aumentada en L3 y persistencia de actividad extraósea en pelvis posterior, post micción.

B. CT pelvis muestra alteraciones secundarias a cirugía. C. Fusión SPECT/CT localiza captación en vejiga posterior, la cual era neurogénica.

La fusión SPECT- Tc ${ }^{99 \mathrm{~m} M D P / C T}$ comprobó que la actividad extraósea correspondía a eliminación urinaria normal del difosfonato.

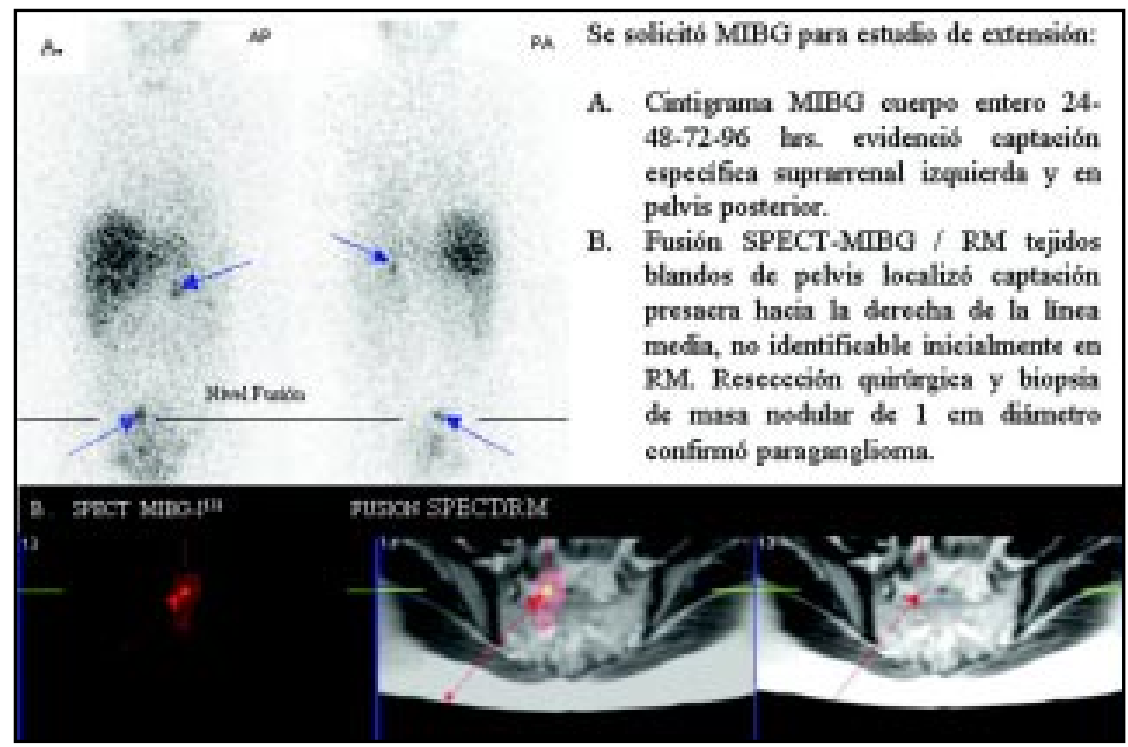

Figura 4. Caso 32. Mujer, 40 años. Feocromocitoma supramenal izquierdo resecado en 1983. Recidiva clínica 2003 con estudio imagenológico negativo. Control 2005 CT y RM detectan nódulo supramenal izquierdo sugerente de feocromocitoma y lesión focal hepática compatible con hemangioma.

Fusión SPECT/RM tejidos blandos de pelvis detecta además del foco supramenal conocido, lesión metastásica presacra, que midió de $1 \mathrm{~cm}$ en la reseccción quirúrgica. Histológicamente correspondió a paraganglioma. 
Tabla 1. Focos que mantuvieron su significado patológico (sp) en la fusión ( $n=37$ )

\begin{tabular}{|c|c|c|c|c|c|c|}
\hline Caso & Región & Focos & & $\begin{array}{l}\text { grama y SPECT inicial } \\
\text { Ubicación }\end{array}$ & & $\begin{array}{l}\text { ión } \\
\text { Ubicación }\end{array}$ \\
\hline 15 & Cráneo & 1 & M & Base cráneo I o encéfalo & M & Tumor encefálico petroclival I \\
\hline 16 & & 1 & M & Base cráneo đmetástasis? & M & Metástasis ósea cóndilo occipital I \\
\hline 24 & & 1 & M & Macizo facial bilateral & M & Lesión neoplásica etmoidoesfenoidal \\
\hline 17 & Cuello & 2 & M & Focos en columna cervical & M & Metástasis C1-C2 y C7 \\
\hline 6 & & 1 & B & Línea media cervical inferior & B & Remanente tiroideo \\
\hline 29 & & 1 & M & Línea media cervical anterior & M & Adenopatías peri y para traqueales \\
\hline 5 & Cervicotorácica & 2 & M & Focos en ápice pulmonar bilateral & M & Micrometástasis mediastino superior \\
\hline 9 & & 1 & M & Masa cervical ¿ganglios infiltrados? & $\mathrm{M}$ & Hueso hiodes y ganglios $<1 \mathrm{~cm}$ \\
\hline 2 & Tórax & 10 & M & Focos pulmonares bilaterales & M & 25 nódulos metastásicos bilaterales \\
\hline 4 & & 1 & B & Foco retroesternal Obs. Timo & B & Timo residual \\
\hline 5 & & 1 & B & Foco retroesternal Obs. Timo & B & Timo residual \\
\hline 7 & & 1 & B & Foco retroesternal Obs. Timo & B & Timo residual \\
\hline 8 & & 2 & M & Focos pulmonares o hiliares I & M & Nódulo Lob inferior I superior $4 \mathrm{~mm}$ \\
\hline 18 & & 1 & B & Foco retroesternal Obs. Timo & B & Timo residual \\
\hline 27 & & 1 & B & Foco retroesternal Obs. Timo & B & Timo residual \\
\hline 30 & & 2 & M & Focos retroesternales altos & $\mathrm{M}$ & Conglomerado mediastino bilateral \\
\hline 33 & & 1 & M & Foco mediastínico infiltrado & M & Esternón sin cambios anatómicos \\
\hline 34 & & 1 & M & Parénquima hepático vs tumor & M & Tejido tumoral hepático \\
\hline 12 & Abdomen & 1 & M & LH D & M & Quistes $2 \mathrm{~cm}$ sgto VII y $3 \mathrm{~cm}$ sgto VIII \\
\hline 23 & & 1 & M & LH D posterior & M & Micrometástasis hepática \\
\hline 28 & & 1 & M & LH D & M & Quiste simple en segmento VI \\
\hline 10 & Pelvis & 1 & B & Foco tejido blando glúteo D & B & Fibroma antiguo en glúteo mayor D \\
\hline 10 & & 1 & B & Vejiga & B & Útero menstrual (cérvix) \\
\hline 32 & & 1 & M & Foco secundario en pelvis posterior & M & Pequeña masa presacra neoplásica \\
\hline
\end{tabular}

M: Maligno. B: Benigno. LH: Lóbulo hepático. D: Derecho. I: Izquierdo.

Tabla 2. Focos que cambiaron de significado patológico (sp) en la fusión ( $n=14$ )

\begin{tabular}{|lllllll|}
\hline & & \multicolumn{3}{c}{ Cintigrama y SPECT inicial } & \multicolumn{2}{l|}{ Fusión } \\
Caso Región & Focos & SP & Ubicación & SP & Ubicación \\
\hline 15 & Cráneo & 1 & M & Obs Apófisis mastoides I & B & Mastoiditis infecciosa I \\
25 & SPN & 1 & M & SPN: Macizo facial I & B & Engrosamiento mucoso seno maxilar I \\
3 & Cervicotorácica & 1 & M & Mediastino superior & B & Inflamación articular esternoclavicular \\
22 & Tórax & 1 & M & Pulmón derecho posterior & B & Secuela retráctil TBC \\
22 & & 1 & M & Hilio pulmonar izquierdo & B & Secuela granulomatosa TBC \\
26 & & 1 & M & Ápice pulmón derecho & B & Secuela retráctil TBC \\
31 & & 1 & B & Obs. Timo & M & Micrometástasis intratoráxica \\
1 & Pelvis & 1 & M & Pelvis posterior & B & Útero menstrual (cérvix) \\
11 & & 1 & M & Fosa ilíaca derecha & B & Quiste ovárico D post aborto espóntaneo \\
13 & 1 & M & Pelvis posterior & B & Utero menstrual (cérvix) \\
14 & 1 & M & Pelvis posterior & B & Eliminación urinaria MDP-Tc99m normal \\
19 & 1 & M & Pelvis posterior & B & Útero menstrual en retroversoflexión \\
20 & 1 & M & Pelvis posterior & B & Útero menstrual (cérvix) \\
21 & 1 & M & Pelvis posterior & B & Utero menstrual con vejiga llena \\
\hline
\end{tabular}

SPN: Senos paranasales. M: Maligno. B: Benigno. D: Derecho. I: Izquierdo. 


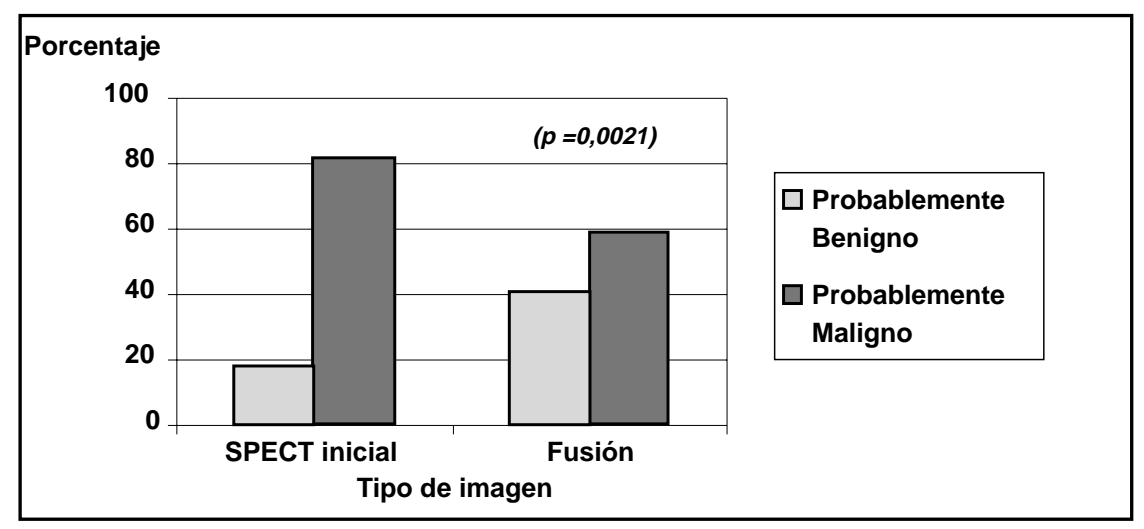

Figura 5. Significado patológico de los focos pre y posfusión.

Una camilla adaptable para gamacámara y tomógrafo habría minimizado la diferencia de posición, al trasladar al paciente entre departamentos con fijaciones y marcas. En vista de su inexistencia recomendamos el uso de $6 \mathrm{ME}$, amortiguando el error movimiento e intensidad de marcación.

Como desventaja, resalta el consumo de tiempo. El protocolo dispuso de una gamacámara doble cabezal, logrando minimizar la duración del SPECT. Respecto al tiempo de procesamiento, éste no sobrepasó al habitual de cintigramas comunes en manos entrenadas, tras cumplirse una curva de aprendizaje, propia de toda nueva técnica.

Respecto al CT-tórax, se podría pensar que quedaría restringido a un estudio localizador, de menor calidad que aquellos con criterio radiológico, pero descartamos esta suposición, ya que a pesar de los artefactos de movimiento respiratorio, observamos lesiones intratorácicas $<1 \mathrm{~cm} \mathrm{y}$ escasa a nula lateralización de las estructuras mediastínicas. A juicio de los autores, el tomógrafo multicorte debiera reservarse para estudios dirigidos reconociendo las ventajas en resolución y el de baja energía para fusión.

La RM, al ser una imagen órgano-dirigida, no presenta estas dificultades, ya que el mismo órgano estudiado actúa como MI, cuya principal desventaja es el costo. La información fisiopatológica entregada depende de los tiempos de relajación (T1, T2), contraste paramagnético usado, contenido grado, perfusión y flujo tumoral entre otros, mientras que en el CT depende del flujo y de la atenuación a los rayos $\mathrm{X}$ por la distinta densidad de los tejidos ${ }^{23,24}$.

La fusión SPECT/RM se realizó en los casos 28, 32 y 34 reemplazando al CT protocolar debido a que en sus diagnósticos particulares (CDT y feocromocitoma) era éticamente correcto aplicar la tecnología disponible con mayor rendimiento en el diagnóstico diferencial de masas hepáticas y tejidos blandos pélvicos $23-26$, como el caso de la Figura 4.

El análisis estadístico tiende a respaldar la conocida alta sensibilidad de los cintigramas tradicionales, a pesar de la relativa carencia de referentes anatómicos, sugiriendo que la fusión estaría justificada sólo en casos seleccionados y no en todo paciente oncológico, ya que el aporte clínico, de ser demostrado histológicamente, alcanzaría a $27 \%$ (14/51 focos).

El 76,5\% de los focos fusionados pertenecieron a pacientes con CDT. Mazzaferri et al, en una importante revisión del tema, encontraron una tasa de recurrencia global de $35 \%$, que justifica los esfuerzos por confirmar el significado de los hallazgos PM de la $\mathrm{ES}^{27}$.

Debido a las caracteństicas físicas del $\mathrm{I}^{131}$, las ES carecen de referentes anatómicos, lo que entorpece la interpretación de focos anormales y varios resultarán en falsos positivos (FP), como los casos comunicados por McDougall et al, explicables por contaminación cutánea, proceso benigno o eliminación urinaria/ gastrointestinal habitual del trazador ${ }^{29-32}$.

Adenopatías, glándulas salivales, infiltración de partes blandas u óseas, son parte de los diagnósti- 
cos diferenciales frente a una ES-I ${ }^{131}$ positiva a nivel cervical (captación asimétrica, persistente, intensa). Al considerar la posibilidad de FP, es importante descartar una sialoadenitis subyacente como efecto colateral del radioyodo. Aunque la fusión es una excelente alternativa, la malignidad puede descartarse con otras imágenes isotópicas de doble fase con MDP-Tc ${ }^{99 m}$ o $\mathrm{Tc}^{99 \mathrm{~m}}$ pertecneciato. El MDP localizaría el foco en una metástasis ósea y el segundo, en una glándula sustituida, práctica aún no incluida en las guías internacionales de $\mathrm{MN}^{28}$.

En mediastino, se ha evidenciado captación $\mathrm{I}^{131}$ en timo fisiológico o hiperplásico por resección quirúrgica, evolución clínica o anatomía al $\mathrm{CT}^{33-36}$. La fusión confirmó esta sospecha en 5 focos de pacientes entre 18 y 31 años con CDT; y la descartó en uno, en quien el foco no correspondía a lesión ni al remanente tímico existente. Este hallazgo permite plantear que el tamaño lesional no parece ser un problema en la metodología propuesta. La radiación dispersa en el SPECT genera imágenes de mayor tamaño al real, explicando la presencia de micrometástasis con anatomía normal en el caso 31. Considerar que las alteraciones funcionales son temporalmente previas a las morfológicas pudiendo presentarse en estructuras anatómicas conservadas.

Frente al antecedente anamnéstico de tuberculosis, granuloma, bronquiectasia o cicatriz pulmonar, la literatura exige descartar un FP en ES positivas para metástasis intratorácica, objetivo logrado al fusionar dichas lesiones con SPECT/CT ${ }^{37-39}$.

Además, los focos hiperémicos en útero menstrual de mujeres con CDT, constituyen una alternativa benigna de actividad de radioyodo en pelvis, no descrita previamente.

Es posible que el mayor aporte de esta experiencia sea el haber identificado a la ES-I ${ }^{131}$ como el cintigrama que más se complementaría con un SPECT y CT segmentario localizador. Los más beneficiados serían los pacientes con $\mathrm{CDT}$, ya que la fusión logró descartar malignidad, reclasificando los focos de mayor captación como proceso benig- no o eliminación del trazador, sin diferenciarlos por niveles de tiroglobulinas plásmáticas $(\mathrm{Tg})$.

Debido a la reciente incorporación de los equipos híbridos PET/CT en Chile, es necesario aclarar al lector no especialista, que el $\mathrm{I}^{131}$ es un trazador específico para identificar tejido tiroideo funcionante in vivo, no aplicable a PET, ya que requiere trazadores emisores de positrones como la fluordoxiglucosa-flúor18, teniendo indicación en casos de CDT con ES negativa y $\mathrm{Tg}>10 \mathrm{ng} / \mathrm{dl}$, criterio ausente en este estudio ${ }^{7}$.

\section{CONCLUSIONES}

La fusión con ME, constituiría una técnica implementable en hospitales que cuenten con medicina nuclear y radiología.

La fusión beneficiaría sólo a pacientes oncológicos seleccionados, cuyo uso racional, respaldará la eficiencia de la técnica.

La fusión SPECT-I ${ }^{131} / \mathrm{CT}$ en CDT entregaría la información anatómica y funcional integrada óptimamente, demostrando su utilidad al caracterizar el significado patológico de hallazgos presuntivamente malignos.

\section{LIMITACIONES}

La metodología presentada carece de seguimiento y confirmación histológica como estándar de oro. Estuvo disponible en un caso, lo que representa la realidad chilena en la práctica clínica respecto a los hallazgos de ES, los que se consideran tratados con radioyodo.

El análisis estadístico está basado en 51 focos de 29 enfermos, muestra lograda en 21 meses. La correcta aplicación del protocolo requería alta supervisión debido a la participación de 2 departamentos de un mismo hospital, lo que influyó en el reclutamiento de más casos. Este bajo número explica la ausencia de conclusiones clínicas respecto a los casos no-CDT. 


\section{REFERENCIAS}

1. Sцомка PJ. Software approach to merging molecular with anatomic information. J Nucl Med 2004; 45 Suppl 1: 36S-45S.

2. Hutton B, Braun M. Software for image registration: Algorithms, accuracy, efficacy. Sem Nucl Med 2003; 33: 180-92.

3. Bocher M, Balan A, Krausz Y, Shrem Y, Lonn A, WILK M ET AL. Gamma camera-mounted anatomical X-ray tomography: technology, system characteristics and first images. Eur J Nucl Med 2000; 27: 619-27.

4. Patton JA, Delbeke D, Sandier MP. Image fusion using an integrated, dual-head coincidence camera with $\mathrm{x}$-ray tube-based attenuation maps. J Nucl Med 2000; 41: 1364-8.

5. Israel O, Keidar Z, Iosilevsky G, Bettman L, Sachs J, FRENKel $A$. The fusion of anatomic and physiologic imaging in the management of patients with cancer. Semin Nucl Med 2001; 31: 191-205.

6. Keidar Z, IsRael O, Krausz Y. SPECT/CT in tumor imaging: technical aspects and clinical applications. Sem Nucl Med 2003; 33: 205-18.

7. MaLk EJ, Bruce DC. Positron-Emission Tomography and Assessment of Cancer Therapy. N Engl J Med 2006; 354: 496-507.

8. Shreve P. Adding structure to function. J Nucl Med 2000; 41: 1380-2.

9. TOWNSEND DW, CheRRY SR. Combining anatomy and function: the path to true image fusion. Eur Radiol 2001; 11: 1968-74 (abstract).

10. Maes F, Colugnon A, Vandermeulen D, Marchal G, SuETENS P. Multimodality image registration by maximization of mutual information. IEEE Trans Med Imaging 1997; 16: 187-98 (abstract).

11. Aqueveque C, González P, Herrmann R, Cereceda M. Primera fusión SPECT Sestamibi y Cardiorresonancia para estudio de viabilidad: caso clínico. Alasbimn Journal 2005; 7: 27. Disponible en: http://www2.alasbimnjournal.cl [Consultado 20 Marzo 2005]

12. Pelosi E, Messa C, Sironi S, Picchio M, Landoni C, Bettinardi V ET al. Value of integrated PET/CT for lesion localization in cancer patients: a comparative study. Eur J Nucl Med Mol Imaging 2004; 31: 932-9.

13. Gutman F, Hangard G, Gardin I, Varmenot N, Pattyn J, Clement JF et al. Evaluation of a Rigid Registration Method of Lung Perfusion SPECT and Thoracic CT. AJR Am J Roentgenol 2005; 185 : 1516-24.

14. Aqueveque C, González P, Díaz JC, Csendes P, JaImovich R, GutiérRez D. Utilidad clínica de la fusión SPECT/ CT en cáncer diferenciado de tiroides. Alasbimn Journal 2005; 7: 27. Disponible en: http://www2.alasbimnjournal.cl [Consultado 20 Marzo 2005].

15. Yamamoto Y, Nishiyama $Y$, Monden T, Matsumura $Y$, Sатон K, ОнкаWA M. Clinical usefulness of fusion of 131I SPECT and CT images in patients with differentiated thyroid carcinoma. J Nucl Med 2003; 44: 1905-10.

16. Maurer CR, Maciunas RJ, Fitzpatrick JM. Registration of head CT images to physical space using a weighted combination of points and surfaces. IEEE Trans Med Imaging 1998; 17: 753-61 (abstract).

17. Tharp K, Israel O, Hausmann J, Bettman L, Martin WH, Daitzchman M et al. Impact of I131 SPECT/ CT images obtained with an integrated system in the follow-up of patients with thyroid carcinoma. Eur J Nucl Med Mol Imaging 2004; 31: 1435-42.

18. Beyer T, Townsend DW, Brun T, Kinahan PE, Charron M, Roddy R et al. A combined PET/CT scanner for clinical oncology. J Nucl Med 2000; 41: 1369-79.

19. Messa C, Bettinardi V, Picchio M, Pelosi E, Landoni C, Gianow L ET al. PET/CT in diagnostic oncology. Q J Nucl Med Mol Imaging 2004; 48: 66-75.

20. SchiшaCi O, SimonetTi G. Fusion imaging in nuclear medicine- applications of dual-modality system in oncology. Cancer Biother Radiopharm 2004; 19: 1-10.

21. Israel O, Yefremov N, Mor M, Haim N, Gaitini D, Epelbaum R ET AL. A new technology of combined transmission (CT) and emission Ga-67 tomography (TET) in the evaluation of patients with lymphoma. J Nucl Med 2000; 41: 70P.

22. Kanematsu M, Kondo H, Goshima S, Kato H, Tsuge U, HIROSE Y ET AL. Imaging liver metastases: Review and update. Eur J Radiol 2006; 58: 217-28.

23. JaMEs W. Reinig Differentiation of Hepatic Lesions with MR Imaging: The Last Word? Radiology 1991; 179: 601-2.

24. Krausz Y, Keidar Z, Kogan I, Even-Sapir E, BarShalom R, Engel A et al. SPECT/CT hybrid imaging with 111In-pentetreotide in assessment of neuroendocrine tumours. Clin Endocrinol (Oxf) 2003; 59: 565-73. 
25. Amthauer H, Ruf J, Bohmg M, López-Hanninen E, ROHLFING T, WeRNECKE KD ET AL. Diagnosis of neuroendocrine tumours by retrospective image fusion: is there a benefit? Eur J Nucl Med Mol Imaging 2004; 31: 342-8.

26. Forster GJ, Laumann C, Nickel O, Kann P, Rueker O, BARTENSTEIN P. SPET/CT image co-registration in the abdomen with a simple and cost-effective tool. Eur J Nucl Med Mol Imaging 2003; 30: 32-9.

27. Mazzaferri E, KLoos R. Current Approaches to Primary Therapy for Papillary and Follicular Thyroid Cancer. JCE \& M 2001; 86: 1447-63.

28. VARIos Autores. Guías de procedimiento de la Sociedad Amenicana de Medicina Nuclear disponibles en http://interactive.snm.org/index.cfm?PageID =772 [Consultado 20 Marzo 2005].

29. McDougaLl IR. Whole-body scintigraphy with radioiodine-131. A comprehensive list of falsepositives with some examples. Clin Nucl Med 1995; 20: 869-75.

30. Ozguven M, Ilgan S, Arslan N, Karacalioglu aO, YUKSEL D, DuNDAR S. Unusual patterns of I-131 contamination. Ann Nucl Med 2004; 18: 271-4.

31. BAKHEET SM, HAMMAMI MM. False-positive radioiodine whole-body scan in thyroid cancer patients due to unrelated pathology. Clin Nucl Med 1994; 19: 325-9.

32. Salvatori M, Saletnich I, Rufini V, Troncone L. Unusual false-positive radioiodine whole-body scans in patients with differentiated thyroid carcinoma. Clin Nucl Med 1997; 22: 380-4.

33. Davidson J, McDougaLl IR. How frequently is the thymus seen on whole-body iodine-131 diagnostic and post-treatment scans? Eur J Nucl Med 2000; 27: 425-30.

34. Wilson LM, BarRington SF, Morrison ID, Kette AG, O'DOHERTY MJ, COAKLEY AJ. Therapeutic implications of thymic uptake of radioiodine in thyroid carcinoma. Eur J Nucl Med 1998; 25: 622-8.

35. Veronikis IE, Simkin P, Braverman LE. Thymic uptake of iodine-131 in the anterior mediastinum. J Nucl Med 1996; 37: 991-2 (abstract).

36. Michigishi T, Mizukami $Y$, Shuke N, Yokoyama K, Noguchi M, Watanabe $Y$ et al. Visualization of the thymus with therapeutic doses of radioiodine in patients with thyroid cancer. Eur J Nucl Med 1993; 20: 75-9.

37. Picolos MK, Habra M, Safdar A, SarLis NJ. Inactive Pulmonary Tuberculosis Mimicking Metastasis from Papillary Thyroid Carcinoma in Diagnostic Radioiodine Whole-Body Scintigraphy. Thyroid 2005; 15: 1105-6.

38. Song HC, Heo YJ, Кім SM, Bom HS. Iodine-131 uptake in focal bronchiectasis mimicking metastatic thymoid cancer. Clin Nucl Med 2003; 28: 351-2.

39. BaKheEt SM, Hammami MM, Powe J, Bazarbashi M, AL SuHAIBANI H. Radioiodine uptake in inactive pulmonary tuberculosis. Eur J Nucl Med 1999; 26: 659-62. 